\title{
Cognitive screening in treatment-naïve HIV-infected individuals in Hong Kong - a single center study
}

Fiona C.C. Chan ${ }^{1}$, Phillip Chan ${ }^{2 *}$ (D), Iris Chan ${ }^{3}$, Andrew Chan', Tommy H. C. Tang ${ }^{1}$, Wilson Lam¹, W. C. Fong ${ }^{1}$, M. P. Lee', Patrick Li ${ }^{4}$ and Germaine H. F. Chan ${ }^{1}$

\begin{abstract}
Background: HIV-associated neurocognitive disorder (HAND) remains prevalent in the era of combination antiretroviral therapy (CART). The prevalence of HAND in Hong Kong is not known.

Methods: Between 2013 and 2015, 98 treatment-naïve HIV-1-infected individuals were referred to and screened by the AIDS Clinical Service, Queen Elizabeth Hospital with (1) the International HIV Dementia Scale (IHDS), a screening tool that targets moderate to severe HAND, (2) the Montreal Cognitive Assessment (MoCA), a frequently used cognitive screening test and (3) the Patient Health Questionnare-9 (PHQ-9), a 9-item questionnaire that evaluates depression symptoms. Within the study period, 57 of them completed the second set of IHDS and MoCA at 6 months after baseline assessment.

Results: Most participants were male (94\%), with a median age of 31 years. At baseline, 38 (39\%) and 25 (26\%) of them scored below the IHDS $(\leq 10)$ and MoCA (25/26) cut-offs respectively. Poor IHDS performers also scored lower on MoCA ( $p=0.039)$ but the correlation between IHDS and MoCA performance was weak $(r=0.29, p=0.004)$. Up to a third of poor IHDS performers (13/38) showed moderate depression (PHQ-9 $>9$ ). In the multivariable analysis, a lower education level $(p=0.088)$, a history of prior psychiatric illness $(p=0.091)$ and the presence of moderate depression $(p=0.079)$ tended to be significantly associated with poor IHDS performance.

At follow-up, 54 out of 57 were on CART, of which 46 (85\%) had achieved viral suppression. Their blood CD4+ T-lymphocytes and IHDS scores were higher at follow-up compared to baseline values (both $p<0.001$ ) but their MoCA performance was similar at both assessments. Of note, 17 participants in this subgroup scored below the IHDS cut-off at both assessments.

Conclusions: Poor IHDS performance, and likely cognitive impairment, was frequently observed in treatment-naïve HIV-infected individuals in our locality. A considerable proportion continued to score below the IHDS cut-off at 6 months after CART. Depression was frequently observed in this vulnerable population and was associated with poor IHDS performance.
\end{abstract}

Keywords: HIV, CART naïve, Cognitive screening, MoCA, IHDS, Hong Kong

\footnotetext{
* Correspondence: phillip.c@searchthailand.org

${ }^{2}$ SEARCH, Thai Red Cross AIDS Research Centre, 104 Ratchadamri Rd,

Khwaeng Pathum Wan, Khet Pathum Wan, Krung Thep Maha Nakhon,

Bangkok 10330, Thailand

Full list of author information is available at the end of the article
}

(c) The Author(s). 2019 Open Access This article is distributed under the terms of the Creative Commons Attribution 4.0 International License (http://creativecommons.org/licenses/by/4.0/), which permits unrestricted use, distribution, and reproduction in any medium, provided you give appropriate credit to the original author(s) and the source, provide a link to the Creative Commons license, and indicate if changes were made. The Creative Commons Public Domain Dedication waiver (http://creativecommons.org/publicdomain/zero/1.0/) applies to the data made available in this article, unless otherwise stated. 


\section{Background}

The introduction of combination antiretroviral therapy (cART) has changed HIV-1 infection from a life-threatening disease to a manageable chronic condition, and life expectancy of people living with HIV (PLWH) is approaching that of non-infected individuals [1]. However, the impact of cART is less significant in the central nervous system (CNS). While CNS opportunistic infections are rare, cognitive impairment remains common in PLWH on cART [2-4]. It is estimated that the frequency of HIV associated dementia (HAD), the most severe form of HIV associated cognitive disorder (HAND), has dropped from 20 to $2 \%$ among PLWH in the cART era. However, the rate of milder forms of HAND still ranges from 15 to $55 \%$ in different cohorts [2-4].

Apart from the impact on work performance and quality of life, cognitive impairment in PLWH could affect drug adherence and hence virological control [5]. In Hong Kong, the population of PLWH exceeded 8000 in 2016 with around 700 new cases reported each year and the prevalence of cognitive impairment in this vulnerable population is largely unknown. In a multi-center cross-sectional study of 10 Asia-Pacific regions in 2008, cognitive impairment was reported in $12 \%$ of 647 clinic-followed PLWH, but this percentage was 23\% among the 61 Hong Kong participants [6]. Estimating the local prevalence of cognitive impairment is essential for health funding, resource allocation and patient support.

This study aimed to estimate the frequency of possible cognitive impairment in cART-naïve HIV-infected individuals referred to HIV-clinic service in Hong Kong. We compared cognitive screening test performance before and after cART and identified potential risk factors that were associated with poor test performance.

\section{Methods}

\section{Study design and participants recruitment}

Participants included all consecutive treatment-naïve referrals to the AIDS Clinical Service, Queen Elizabeth Hospital, Hong Kong, between October 1, 2013 and October 31, 2015. Only ethnic Chinese were included to ensure standardization of population and the cognitive screening procedures. Individuals with a known history of major medical or neurological disorders were excluded. At enrollment, the participants underwent clinical assessment, cognitive and depression screening, and laboratory tests. They were reassessed approximately six months later. All participants received written information sheets for the study and provided verbal informed consent. This study was approved by the Kowloon Central/Kowloon East Research Ethics Committee of Hospital Authority, Hong Kong (Reference number: KC/KE-14-0065/FR-1).
Clinical and laboratory data acquisition

At baseline, demographic data, social history and past medical history, including route of HIV-1 transmission, were collected. Laboratory tests included blood CD4+ T-lymphocytes level, HIV-1 subtyping, HIV-1 ribonucleic acid (RNA) quantification, and serology of syphilis and viral hepatitis (B and $\mathrm{C}$ ). Blood CD4+ T-lymphocytes and HIV-1 RNA levels were tested again in parallel with cognitive screening at follow-up. Individual cART regimens were recorded based on the CNS penetration-effectiveness (CPE) index [7]: a high CNS penetrating regime was defined as $\mathrm{CPE}>7$.

\section{Cognitive function and depression assessment}

A formal diagnosis of HAND relies on detailed neuropsychiatric and functional status assessment conducted by a clinical psychologist (CP) [3] but such assessments require a significant amount of time and resources. The International HIV dementia scale (IHDS) is a validated brief 3-min screening test that is ideal for this pilot study which aims to estimate the frequency of cognitive impairment in newly referred patients. IHDS examines motor speed, psychomotor speed and memory-recall function [8] and a score of $\leq 10$ (out of 12) shows a reasonable sensitivity (64-74\%) and specificity (55-66\%) in identifying moderate to severe HAND [9]. The Hong Kong version of the Montreal Cognitive Assessment (MoCA) was added to supplement the breadth of cognitive screening (visuospatial/executive, naming, memory, attention, language, abstraction, delayed recall, and orientation). In our locality, a cut-off of $21 / 22$ (out of 30 ) has been validated for evaluating mild cognitive impairment in elderly and stroke patients $[10,11]$, while a cut-off of 25/26 is used in the original English version and previous HAND-related studies in Asian societies including Singapore [12] and Korea [13, 14]. Both cut-off values were included in the results for reference.

At both baseline and follow-up assessments, participants were screened with IHDS and MoCA by CP or trained research assistants. At baseline, participants also completed the Patient Health Questionnare-9 (PHQ-9), a 9-item questionnaire which scores each of the 9 DSM-IV criteria from "0" (not at all) to "3" (nearly every day) over the previous 2 weeks [15]. The Chinese version of PHQ-9 has been validated in Hong Kong [16], with a cut-off score $>9$ for moderate depression [15].

\section{Statistical analysis}

Continuous variables were presented as median and interquartile ranges (IQR). Pearson's Chi-squared test and Fisher's exact test were used for categorical variables. Non-parametric Mann-Whitney U test was used for continuous variables. Multivariable logistic regression models were fitted to identify baseline factors associated 
with poor IHDS performance (cut-off: < 10). McNemar and Wilcoxon signed-rank test were used to compare variables before and after cART accordingly. A $p$-value $<0.05$ was considered significant. Statistical analyses were performed using SPSS Version 20.0 (International Business Machines Corporation, New York, USA).

\section{Results}

During the study period, 102 patients were referred, of whom 4 individuals were excluded from participation: two were cART-experienced, one had a CNS opportunistic infection, and one had intellectual disability. None were lost to follow-up but one participant succumbed before follow-up due to a non-HIV-related illness. Fifty-seven completed the follow-up assessment within the study period.

Of the 98 enrolled individuals (Table 1), more than a third ( $n=37,38 \%)$ were referred from our in-patient service for continuation of care. Most were male $(n=92,94 \%)$ with a median age of 31 years (IQR 26-43). Sexual contact among men who have sex with men (MSM) was the predominant route of HIV acquisition (77\%). HIV-1 subtypes CRF01_AE (42\%) and B (37\%) were the predominant strains. Eighteen (18\%) had a previous AIDS-defining illness. The median pre-cART blood HIV-1 RNA level was 5.07 (IQR 4.68-5.47) $\log _{10}$ copies $/ \mathrm{mL}$ and the median nadir CD4+ T-lymphocyte level was 270 (IQR 106-376) cells/ $\mu \mathrm{L}$.

\section{Risk factors of poor IHDS performance at baseline}

At baseline, 38 participants (39\%) scored $\leq 10$ on IHDS. Their demographic and clinical characteristics were compared to those who scored above this cut-off (Table 2). In the univariable analyses, poor IHDS performers had higher rates of prior psychiatric illness $(24 \%$ vs. $8 \%, p=0.034)$ and moderate depression (PHQ-9 > 9) (34\% vs. $17 \%, p=0.048)$. They tended to have lower education level ( $40 \%$ vs. $57 \%$ tertiary education, $p=0.097$ ) and blood CD4+ T-lymphocyte counts (241 (IQR 69-320) vs. 298 (IQR 160-400), $p=0.078$ ). Age, sex, smoking, alcohol and substance use, blood HIV-1 RNA level, syphilis, and hepatitis C co-infection did not associate with poor IHDS performance (all $p>$ 0.1 ). In the multivariable analysis, prior psychiatric illness (adjusted odds ratio (aOR) 2.99; 95\% CI 0.84-10.66, $p=$ 0.091), moderate depression (aOR 2.52; 95\% CI 0.90-7.06, $p=0.079$ ) and lower education level (tertiary education: aOR 0.47 ; 95\%CI $0.20-1.17, p=0.088$ ) tended to be significantly associated with poor IHDS performance, while CD4+ T-lymphocyte level was no longer significant.

\section{Correlation between IHDS and MoCA performance at baseline}

Poor IHDS performers also performed worse on the MoCA compared to those who scored above the IHDS
Table 1 Demographic and Clinical Characteristics of Participants at Baseline $(N=98)$

\begin{tabular}{|c|c|}
\hline Male, $n(\%)$ & $92(94)$ \\
\hline Female, $n(\%)$ & $6(6)$ \\
\hline Age, year & $31(26-43)$ \\
\hline Tertiary education, $n$ (\%) & $49(50)$ \\
\hline Current Smoker, $n$ (\%) & $31(32)$ \\
\hline Current or ex-drinker, $n(\%)$ & $22(22)$ \\
\hline History of substance Use, $n$ (\%) & $41(42)$ \\
\hline Prior psychiatric illness, $n$ (\%) & $14(14)$ \\
\hline \multicolumn{2}{|l|}{ Route of transmission, $n(\%)$} \\
\hline MSM & $75(77)$ \\
\hline Other & $23(23)$ \\
\hline \multicolumn{2}{|l|}{ HIV-1 subtype, $n$ (\%) } \\
\hline CRF01_AE & $41(42)$ \\
\hline B & $36(37)$ \\
\hline Other & $21(21)$ \\
\hline AIDS $^{\mathrm{a}}, n(\%)$ & $18(18)$ \\
\hline Blood CD4+ T-cells nadir ${ }^{b}$ (cells/ $\mu \mathrm{L}$ ) & $270(106-376)$ \\
\hline Blood HIV-1 RNA, log10 copies/ml & $5.07(4.68-5.47)$ \\
\hline Hepatitis B virus co-infection, $n(\%)$ & $6(6)$ \\
\hline Hepatitis C virus co-infection, $n(\%)$ & $7(7)$ \\
\hline Syphilis co-infection, $n$ (\%) & $40(41)$ \\
\hline Recent in-patient care, $n(\%)$ & $37(38)$ \\
\hline IHDS $\leq 10, n(\%)$ & $38(39)$ \\
\hline MoCA $\leq 25, n(\%)$ & $25(26)$ \\
\hline MoCA $\leq 21, n(\%)$ & $8(8)$ \\
\hline MoCA score & $27(25-28)$ \\
\hline Moderate Depression (PHQ-9 > 9),$n(\%)$ & $23(24)$ \\
\hline
\end{tabular}

Median (IQR) is presented unless specified otherwise

a Defined by the presence of AIDS-defining illness regardless of CD4+ T-lymphocyte levels

${ }^{\mathrm{b}} n=96$

Abbreviations: IHDS International HIV Dementia Scale, MoCA Montreal Cognitive Assessment, PHQ-9 Patient Health Questionnare-9, MSM men who have sex with men

cut-off (27 (IQR 24-28) vs. 28 (IQR 26-29), $p=0.039$ ). Participants' IHDS scores were only weakly correlated with their MoCA performance (Bivariate correlation (Spearman's): $\mathrm{r}=0.29, p=0.004)$. In particular, nearly one third $(n=24)$ of the 73 participants who scored above the MoCA cut-off ( $>25)$ scored below the IHDS cut-off. The participants' IHDS and MoCA scores are illustrated in Fig. 1.

\section{Clinical and cognitive screening outcomes at 6 months follow-up}

Within the study period, 57 participants completed their second assessment, approximately 6 months after the baseline assessment. Compared to those who did not yet 
Table 2 Factor Associated with Cognitive Impairment Status According to IHDS Score

\begin{tabular}{|c|c|c|c|c|c|c|}
\hline & & & Univariable Analy & & Multivariable Analysis & \\
\hline & $\begin{array}{l}\text { Non-impaired } \\
(\mathrm{IHDS}>10) \\
(n=60)\end{array}$ & $\begin{array}{l}\text { Impaired } \\
(\mathrm{IHDS} \leq 10) \\
(n=38)\end{array}$ & $\begin{array}{l}\text { Odds Ratio } \\
(95 \% \mathrm{Cl})\end{array}$ & $p$-value & $\begin{array}{l}\text { Adjusted Odds Ratio } \\
(95 \% \mathrm{Cl})\end{array}$ & $p$-value \\
\hline áFemale sex, n (\%) & $3(5)$ & $3(8)$ & $1.63(0.31-8.52)$ & 0.674 & & \\
\hline Age, year & $31(25-40)$ & $32(26-45)$ & & 0.358 & & \\
\hline${ }^{\mathrm{b}}$ Tertiary education, $n$ (\%) & $34(57)$ & $15(40)$ & $0.50(0.22-1.14)$ & 0.097 & $0.47(0.20-1.17)$ & 0.088 \\
\hline${ }^{\mathrm{b} C}$ Current smoker, $n(\%)$ & $19(32)$ & $12(32)$ & $1.00(0.44-2.25)$ & 1.000 & & \\
\hline${ }^{\mathrm{b}}$ Current or ex-drinker, $n(\%)$ & $14(23)$ & $8(21)$ & $0.88(0.33-2.34)$ & 0.792 & & \\
\hline bHistory of substance use, $n$ (\%) & $23(38)$ & $18(47)$ & $1.45(0.64-3.30)$ & 0.377 & & \\
\hline${ }^{b}$ Prior psychiatric illness, $n$ (\%) & $5(8)$ & $9(24)$ & $3.41(1.05-11.13)$ & 0.034 & $2.99(0.84-10.66)$ & 0.091 \\
\hline Blood HIV-1 RNA, $\log _{10}$ copies/ml & $5.01(4.61-5.39)$ & $5.11(4.81-5.75)$ & & 0.139 & & \\
\hline CD4+ T-cells nadir (cells/ $\mu \mathrm{L}$ ) & $298(160-400)$ & $241(69-320)$ & & 0.078 & & NS \\
\hline${ }^{\mathrm{b}} \mathrm{CD} 4+\mathrm{T}$-cells nadir $<200$ cells/ $\mu \mathrm{L}, n(\%)$ & $18(30)$ & $13(34)$ & $1.21(0.51-2.89)$ & 0.662 & & \\
\hline aHepatitis C virus co-infection, $n(\%)$ & $5(8)$ & $2(5)$ & $0.61(0.11-3.32)$ & 0.703 & & \\
\hline b,c Syphilis co-infection, n (\%) & $22(37)$ & $18(47)$ & $1.56(0.68-3.55)$ & 0.294 & & \\
\hline dPHQ-9 score & $5(2-8)$ & $8(4-12)$ & & 0.127 & & \\
\hline b,d Moderate depression (PHQ-9 > 9), $n$ (\%) & $10(17)$ & $13(34)$ & $2.67(0.99-7.17)$ & 0.048 & $2.52(0.90-7.06)$ & 0.079 \\
\hline
\end{tabular}

Median (IQR) is presented unless specified otherwise

${ }^{\text {a}}$ Fisher's Exact test

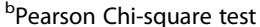

'History of infection (enzyme immunoassay (EIA)-Treponemal pallidum assay (TPA) positivity)

${ }^{d} n=96$

Abbreviations: IHDS International HIV Dementia Scale, MoCA Montreal Cognitive Assessment, PHQ-9 Patient Health Questionnare-9, NS Not significant

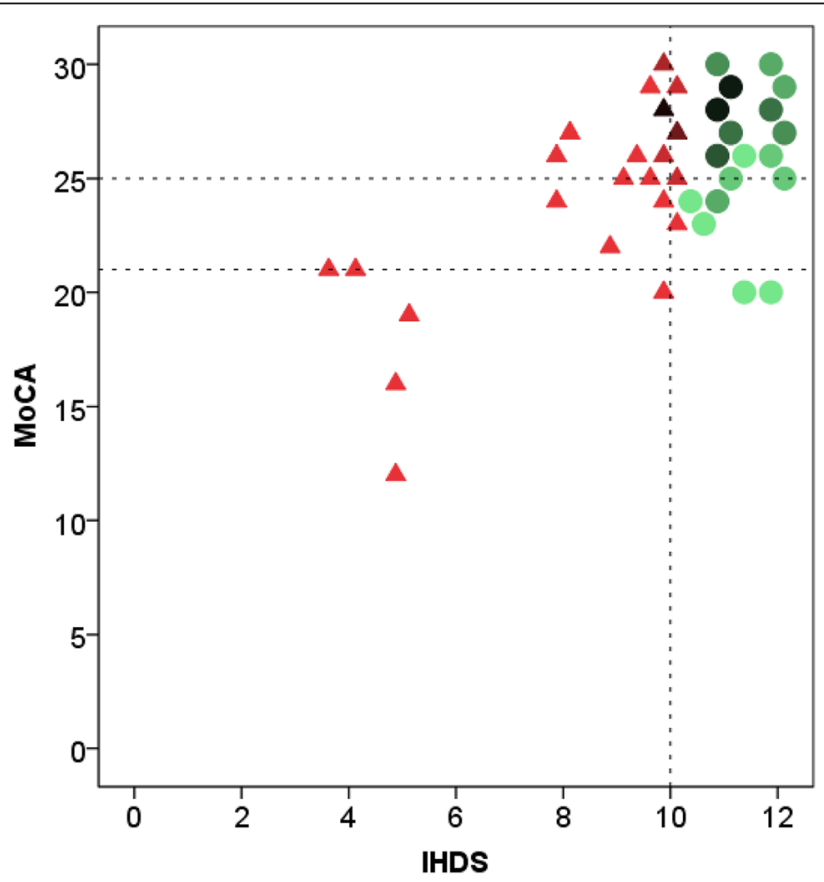

IHDS $<=10$

No

$\triangle$ Yes

\section{Scale}

8

7

(6)

4

3

2

Fig. 1 Participants' Performance in IHDS and MoCA at Baseline. Dotted lines: cut-offs of MoCA $(\leq 21$ and $\leq 25)$ and IHDS $(\leq 10)$ 
complete the second assessment, this subgroup of participants had a lower baseline IHDS score (10 (IQR $10-11$ ) vs. 11 (IQR $11-12$ ), $p<0.001$ ). They also had a high proportion of poor IHDS performers (58\% vs $12 \%, p<0.001)$. However, both subgroups were statistically similar in terms of demographic and clinical parameters except that the subgroup with follow-up had a higher blood HIV-1 RNA at baseline (5.11 vs $4.91 \log _{10}$ copies/ $\mathrm{ml}, p=0.010$ ) (Additional file 1: Table S1).

Fifty-four of the 57 participants who completed both assessments were on cART (Table 3) and 46 (85\%) had achieved viral suppression at follow-up (blood HIV-1 RNA $<20$ copies/ml). Their blood CD4+ T-lymphocyte level and IHDS performance both improved compared to baseline (468 (IQR 261-673) vs. 248 (IQR46-355) cells $/ \mathrm{mm} 3, \mathrm{p}<0.001$ and 11 (IQR $10-12$ ) vs. 10 (IQR $10-11), p<0.001$ respectively). None of them converted from above to below the IHDS cut-off at follow-up and their MoCA test performances were statistically similar between two assessments $(p>0.1)$. Seventeen out of thirty-one participants who scored below the IHDS cut-off at baseline again scored below the cut-off at follow-up.

Linear regression was employed to determine factors that were associated with the change in IHDS scores (i.e. follow-up IHDS score minus baseline IHDS score) (Additional file 2: Table S2). In the univariable analysis, older age was associated with IHDS improvement (Mean difference 0.03, 95\% CI (0.01 to 0.06), $p=0.013$ ). Tertiary education and history of prior psychiatric illness, which tended to be significantly associated with IHDS performance at baseline, were not associated with IHDS change $(p>0.1)$. HIV-related factors, including plasma CD4+ T-lymphocytes nadir, viral suppression status, and CPE index of cART were not associated with change of IHDS scores $(p>0.1)$.

Table 3 Clinical and Cognitive Outcomes of the Subset of Participants with Follow-up $(n=54)^{\text {a }}$

\begin{tabular}{llll}
\hline & Baseline & Follow-up & $p$-value \\
\hline CD4+ T-lymphocyte (cells/ $\mu$ L) & $248(46-355)$ & $468(261-673)$ & $<0.001$ \\
On cART with CPE $>7, n(\%)$ & - & $7(13)$ & - \\
HIV-1 RNA suppression ${ }^{b}, n(\%)$ & - & $46(85)$ & - \\
IHDS score & $10(10-11)$ & $11(10-12)$ & $<0.001$ \\
IHDS $\leq 10, n(\%)$ & $31(57)$ & $17(32)$ & $<0.001$ \\
MoCA score & $27(26-29)$ & $27(26-28)$ & 0.818 \\
MoCA $\leq 25, n(\%)$ & $13(24)$ & $12(22)$ & 1.000 \\
MoCA $\leq 21, n(\%)$ & $4(7)$ & $4(7)$ & 1.000 \\
\hline
\end{tabular}

Median (IQR) is presented unless specified otherwise

a Only included participants who were on CART

${ }^{\mathrm{b}}$ Blood HIV-1 RNA level $<20$ copies $/ \mathrm{mL}$

Abbreviations: IHDS International HIV Dementia Scale, MoCA Montreal

Cognitive Assessment, CPE CNS penetration-effectiveness

\section{Discussion}

This study estimated the frequency of possible cognitive impairment based on an IHDS cut-off $(\leq 10)$ that targets more severe forms of HAND. Recent studies suggest a higher cut-off of $\leq 11$ to improve the IHDS sensitivity towards milder forms of HAND $[17,18]$. At baseline, up to $40 \%$ of this group of relatively young, male predominant, cART-naïve individuals scored below the IHDS cut-off. In the MoCA test that examined a different set of cognitive domains, 26 and $8 \%$ scored below the original English version (25/26) and locally validated $(21 / 22)$ cut-offs, respectively. Of note, six participants scored below both IHDS and MoCA 21/22 cut-offs, suggesting multi-domain cognitive impairment. Poor IHDS performers also scored lower on the MoCA but the correlation was weak. Up to a third of participants with normal MoCA scores (>25) scored below the IHDS cut-off, suggesting a discrete impairment in motor, psychomotor and memory-recall functions without a major involvement in cognitive domains examined by MoCA. Thus, a stand-alone MoCA test would be insufficient for cognitive screening in this group of individuals.

The subgroup of participants who completed the second cognitive screening within the study period had poorer IHDS performance and higher blood HIV-1 RNA levels than the remaining participants while their MoCA performance and clinical parameters were similar. The higher rate of poorer IHDS performers in this subgroup seems random, rather than driven by HIV-1 RNA level, as HIV-1 RNA was not associated with IHDS performance in the multivariable analysis at baseline.

At follow-up, this subset of participants showed an improvement in IHDS performance after cART, but the increment in score was modest. Moreover, a considerable proportion of these participants again scored below the IHDS cut-off at follow-up. Improvement in MoCA test performance was also absent. The overall lack of improvement in both tests could be due to irreversible neurocognitive impairment, driven by HIV-1 infection and/or other etiologies. Other possibilities include the insensitivity of IHDS and MoCA to detect cognitive improvement or a delayed onset of cognitive improvement up to 9 months after cART $[19,20]$.

At baseline, education, prior psychiatric illness and co-existing moderate depression (PHQ-9>9), but not HIV-specific parameters (HIV-1 RNA level and CD4+ T-lymphocytes nadir) $[2,4,21]$ or co-infections (syphilis and HCV) $[22,23]$, tended to be independently associated with poor IHDS performance. The association between IHDS performance and education, particularly years of education, was previously highlighted in another study [17]. Better education is generally considered as a protective factor against cognitive impairment, likely due 
to a better cognitive reserve that contributes to resilience against neuropathological insults [24].

The association between mood disorders and cognitive impairment is frequently reported. In particular, major psychiatric illnesses including depression are linked to long term structural brain changes [25] and cognitive decline [26]. Depression is frequently observed in HIV-infected populations [27] and is also a risk factor for HAND [21]. Differentiating the effect of depression from HAND in neurocognitive assessment is challenging and requires detailed neuropsychiatric assessment [28]. However, none of the factors that were associated with baseline IHDS performance nor HIV-related parameters or CPE index of cART were associated with the change of IHDS performance after cART. This general lack of association could be related to the aforementioned irreversible cognitive impairment, insensitivity of the IHDS, or brief interval between assessments for observing the benefit of cART on neurocognitive functioning.

We observed poor IHDS performance in 39\% of treatment-naïv HIV-infected study participants, a frequency based on a cut-off that targets for more severe forms of HAND. Despite the usefulness of MoCA in degenerative neurocognitive diseases, $\mathrm{MoCA}$ alone may not be an ideal screening tool in HIV-infected populations because of the limited correlation with IHDS outcomes. A considerable proportion of our participants had concomitant moderate depression symptoms (PHQ-9 > 9), which tended to be independently associated with poor IHDS performance. Despite cART resulting in virological control in the majority of the group with follow-up, poor IHDS performance persisted in a sizable proportion of them. The findings highlight the need for comprehensive allied health support in contemporary HIV care, including cognitive and mood assessment, and cognitive rehabilitation may be needed. Hong Kong has a relatively young HIV-infected population with a median age under 40. As this population of PLWH ages due to the improved survival and an as yet stable number of newly reported cases, the service demand for cognitive and mood disorders is expected to increase.

Our study has its limitations. First, although the co-dominant HIV-1 B and CRF_01AE subtypes in our participants is compatible with the local HIV-1 strains pattern [29], they had a relatively high rate of recent hospitalization and might not fully match the new local HIV-1 cases in terms of disease severity. The frequency of AIDS in this study was $18 \%$, compared to $14 \%$ (218/ 1558 ) among total newly reported cases for the corresponding period in Hong Kong (HIV Surveillance Report 2014 and 2015, Centre for Health Protection, Department of Health). Second, repeating cognitive tests at 6 months after cART could be too early to observe cognitive improvement $[19,20]$. Third, the higher rate of poorer IHDS performers at baseline among the subgroup with follow-up assessment may lead to overestimation of the frequency of persistent cognitive impairment after cART. Lastly, cognitive outcomes in this study were estimated by IHDS and MoCA, which are designed for screening purposes. The lack of HIV-negative controls limits the tests' validity in estimating the frequency of cognitive impairment of the study participants.

\section{Conclusion}

Our study suggests that moderate to severe cognitive impairment exists in a considerable proportion of treatment-naïve PLWH in Hong Kong. The population of PLWH in Hong Kong is relatively young and is going to expand further. Our findings support the need for implementing cognitive and mood disorder assessments in routine HIV clinical care. This approach may reduce longer term neurocognitive impairment and alleviate its socioeconomic consequences.

\section{Additional files}

Additional file 1: Table S1. Demographic and Clinical Characteristics of Participants with and without Second Cognitive Screening. (DOCX 15 kb)

Additional file 2: Table S2. Factor Correlation with IHDS Changes. (DOCX $14 \mathrm{~kb})$

\section{Acknowledgements}

We thank Drs. Eugene Kroon and Donn Colby for the helpful discussions and their advice, and the Departments of Medicine and Clinical Psychology of Queen Elizabeth Hospital, Hong Kong for supporting this study.

\section{Funding}

The authors declare that there is no additional funding received beyond routine clinical care for the research participants.

Availability of data and materials

The datasets used and/or analyzed during the current study are available from the corresponding author on reasonable request.

\section{Authors' contributions}

Study Design: CC, IC, AC, ML. Data Acquisition: CC. Cognitive and Depression screening: IC. Statistical analysis: CC, PC, WF, GC. Out-patient clinic follow-up for HIV: TT, WL, ML, PL. Manuscript preparation: CC, PC, IC, AC, TT, WL, WF, $\mathrm{ML}, \mathrm{PL}, \mathrm{GC}$. All authors have read and approved the manuscript.

Ethics approval and consent to participate

All participants received written information sheets and provided verbal informed consent in this study. It aimed to minimize the number of document to protect participants' personal information. It is a common practice locally and the method was approved by the Kowloon Central / Kowloon East Research Ethics Committee of Hospital Authority (Reference number: KC/KE-14-0065/FR-1).

Consent for publication

Not applicable.

Competing interests

The authors declare that they have no competing interests. 


\section{Publisher's Note}

Springer Nature remains neutral with regard to jurisdictional claims in published maps and institutional affiliations.

\section{Author details}

'Department of Medicine, Queen Elizabeth Hospital, Hong Kong, SAR, People's Republic of China. ${ }^{2}$ SEARCH, Thai Red Cross AIDS Research Centre, 104 Ratchadamri Rd, Khwaeng Pathum Wan, Khet Pathum Wan, Krung Thep Maha Nakhon, Bangkok 10330, Thailand. ${ }^{3}$ Department of Clinical Psychology, Queen Elizabeth Hospital, Hong Kong, SAR, People's Republic of China. ${ }^{4}$ Hong Kong Sanatorium and Hospital, Hong Kong, SAR, People's Republic of China.

Received: 4 June 2018 Accepted: 6 February 2019

Published online: 13 February 2019

\section{References}

1. Samji H, Cescon A, Hogg RS, Modur SP, Althoff KN, Buchacz K, Burchell AN, Cohen M, Gebo KA, Gill MJ, et al. Closing the gap: increases in life expectancy among treated HIV-positive individuals in the United States and Canada. PLoS One. 2013;8(12):e81355.

2. Heaton RK, Clifford DB, Franklin DR Jr, Woods SP, Ake C, Vaida F, Ellis RJ, Letendre SL, Marcotte TD, Atkinson JH, et al. HIV-associated neurocognitive disorders persist in the era of potent antiretroviral therapy: CHARTER study. Neurology. 2010;75(23):2087-96

3. Antinori A, Arendt G, Becker JT, Brew BJ, Byrd DA, Cherner M, Clifford DB, Cinque P, Epstein LG, Goodkin K, et al. Updated research nosology for HIVassociated neurocognitive disorders. Neurology. 2007;69(18):1789-99.

4. Robertson KR, Smurzynski M, Parsons TD, Wu K, Bosch RJ, Wu J, McArthur JC, Collier AC, Evans SR, Ellis RJ. The prevalence and incidence of neurocognitive impairment in the HAART era. AIDS. 2007;21(14):1915-21.

5. Anand P, Springer SA, Copenhaver MM, Altice FL. Neurocognitive impairment and HIV risk factors: a reciprocal relationship. AIDS Behav. 2010; 14(6):1213-26

6. Wright E, Brew B, Arayawichanont A, Robertson K, Samintharapanya K, Kongsaengdao S, Lim M, Vonthanak S, Lal L, Sarim C, et al. Neurologic disorders are prevalent in HIV-positive outpatients in the Asia-Pacific region. Neurology. 2008;71(1):50-6

7. Letendre SL, Ellis RJ, Everall I, Ances B, Bharti A, McCutchan JA. Neurologic complications of HIV disease and their treatment. Top HIV Med. 2009;17(2): 46-56.

8. Mind Exchange Working G. Assessment, diagnosis, and treatment of HIVassociated neurocognitive disorder: a consensus report of the mind exchange program. Clin Infect Dis. 2013;56(7):1004-17.

9. Haddow LJ, Floyd S, Copas A, Gilson RJ. A systematic review of the screening accuracy of the HIV dementia scale and international HIV dementia scale. PLoS One. 2013;8(4):e61826.

10. Wong A, Law LS, Liu W, Wang Z, Lo ES, Lau A, Wong LK, Mok VC. Montreal cognitive assessment: one cutoff never fits all. Stroke. 2015;46(12):3547-50.

11. Yeung PY, Wong LL, Chan CC, Leung JL, Yung CY. A validation study of the Hong Kong version of Montreal cognitive assessment (HK-MoCA) in Chinese older adults in Hong Kong. Hong Kong Med J. 2014;20(6):504-10.

12. Chan LG, Kandiah N, Chua A. HIV-associated neurocognitive disorders (HAND) in a south Asian population - contextual application of the 2007 criteria. BMJ Open. 2012;2(1):e000662.

13. Kim WJ, Ku NS, Lee YJ, Ahn JY, Kim SB, Ahn HW, Hong KW, Song JY, Cheong HJ, Kim WJ, et al. Utility of the Montreal cognitive assessment (MoCA) and its subset in HIV-associated neurocognitive disorder (HAND) screening. J Psychosom Res. 2016;80:53-7.

14. Ku NS, Lee Y, Ahn JY, Song JE, Kim MH, Kim SB, Jeong SJ, Hong KW, Kim E, Han SH, et al. HIV-associated neurocognitive disorder in HIV-infected Koreans: the Korean NeuroAIDS project. HIV Med. 2014;15(8):470-7.

15. Kroenke K, Spitzer RL, Williams JB. The PHQ-9: validity of a brief depression severity measure. J Gen Intern Med. 2001;16(9):606-13.

16. Yu X, Tam WW, Wong PT, Lam TH, Stewart SM. The patient health Questionnaire-9 for measuring depressive symptoms among the general population in Hong Kong. Compr Psychiatry. 2012;53(1):95-102.

17. de Almeida SM, Kamat R, Cherner M, Umlauf A, Ribeiro CE, de Pereira AP, Franklin D, Heaton RK, Ellis RJ. Improving detection of HIV-associated cognitive impairment: comparison of the international HIV dementia scale and a brief screening battery. J Acquir Immune Defic Syndr. 2017;74(3): 332-8.

18. Rodrigues RA, Oliveira RL, Grinsztejn B, Silva MT. Validity of the international HIV dementia scale in Brazil. Arq Neuropsiquiatr. 2013;71(6):376-9.

19. Cysique LA, Vaida F, Letendre S, Gibson S, Cherner M, Woods SP, McCutchan JA, Heaton RK, Ellis RJ. Dynamics of cognitive change in impaired HIV-positive patients initiating antiretroviral therapy. Neurology. 2009;73(5):342-8.

20. Tozzi V, Narciso P, Galgani S, Sette P, Balestra P, Gerace C, Pau FM, Pigorini F, Volpini V, Camporiondo MP, et al. Effects of zidovudine in 30 patients with mild to end-stage AIDS dementia complex. AIDS. 1993;7(5):683-92.

21. Saylor D, Dickens AM, Sacktor N, Haughey N, Slusher B, Pletnikov M Mankowski JL, Brown A, Volsky DJ, McArthur JC. HIV-associated neurocognitive disorder--pathogenesis and prospects for treatment. Nat Rev Neurol. 2016:12(4):234-48.

22. Vivithanaporn P, Nelles K, DeBlock L, Newman SC, Gill MJ, Power C. Hepatitis $C$ virus co-infection increases neurocognitive impairment severity and risk of death in treated HIV/AIDS. J Neurol Sci. 2012;312(1-2):45-51.

23. Marra CM, Deutsch R, Collier AC, Morgello S, Letendre S, Clifford D, Gelman B, McArthur J, McCutchan JA, Simpson DM, et al. Neurocognitive impairment in HIV-infected individuals with previous syphilis. Int J STD AIDS 2013;24(5):351-5.

24. Stern $Y$. What is cognitive reserve? Theory and research application of the reserve concept. J Int Neuropsychol Soc. 2002;8(3):448-60.

25. Lorenzetti V, Allen NB, Fornito A, Yucel M. Structural brain abnormalities in major depressive disorder: a selective review of recent MRI studies. J Affect Disord. 2009;117(1-2):1-17.

26. Millan MJ, Agid Y, Brune M, Bullmore ET, Carter CS, Clayton NS, Connor R, Davis S, Deakin B, DeRubeis RJ, et al. Cognitive dysfunction in psychiatric disorders: characteristics, causes and the quest for improved therapy. Nat Rev Drug Discov. 2012;11(2):141-68.

27. Nanni MG, Caruso R, Mitchell AJ, Meggiolaro E, Grassi L. Depression in HIV infected patients: a review. Curr Psychiatry Rep. 2015;17(1):530.

28. Cysique LA, Deutsch R, Atkinson JH, Young C, Marcotte TD, Dawson L, Grant I, Heaton RK, GrouP H. Incident major depression does not affect neuropsychological functioning in HIV-infected men. J Int Neuropsychol Soc. 2007:13(1):1-11.

29. Chen JH, Wong KH, Chen Z, Chan K, Lam HY, To SW, Cheng VC, Yuen KY, Yam WC. Increased genetic diversity of HIV-1 circulating in Hong Kong. PLoS One. 2010;5(8):e12198.

\section{Ready to submit your research? Choose BMC and benefit from:}

- fast, convenient online submission

- thorough peer review by experienced researchers in your field

- rapid publication on acceptance

- support for research data, including large and complex data types

- gold Open Access which fosters wider collaboration and increased citations

- maximum visibility for your research: over $100 \mathrm{M}$ website views per year

At BMC, research is always in progress.

Learn more biomedcentral.com/submissions 